\title{
HRaidTools: An On-line Suite of Simulation Tools for Heterogeneous RAID Systems
}

\author{
J. L. Gonzalez \\ Cd. Valles Institute of \\ Technology, S.L.P., Mexico \\ joseluig@ac.upc.edu
}

\author{
Toni Cortes \\ Department of Computer \\ Architecture and Barcelona \\ Supercomputing Center, Spain \\ toni@ac.upc.edu \\ Ana Maria Piedad-Rubio \\ Cd. Valles Institute of \\ Technology \\ ampiedad@itcdvalles.edu.mx
}

\author{
Jaime Delgado-Meraz \\ Cd. Valles Institute of \\ Technology, S.L.P., Mexico \\ j2deme@gmail.com
}

\begin{abstract}
This work presents HRaidTools, an on-line suite of tools for simulating Heterogeneous RAID systems, which includes tools such as a RAID-designer, an I/O synthetic workload generator, a friendly simulation launcher and results collector. This suite has been designed to reduce or even eliminate the errors in simulations produced by the humans and the problems arise when using the simulator in different environments. In addition, HRaidTools also includes an Adaptive Collaborative Work architecture based on profiles of social networks, which have been designed for handling and sharing simulation contents such as experiments, configurations, workloads, results and graphs while following the common hierarchies in research groups. Finally, this suite includes several heterogeneous data distribution policies and redundancy levels unavailable in current RAID simulators.
\end{abstract}

\section{Categories and Subject Descriptors}

H.4 [Information Systems Applications]: Miscellaneous

\section{General Terms}

Simulation Tools

\section{Keywords}

RAID, Heterogeneity, Collaborative Work

\section{INTRODUCTION}

RAID systems [8] are the most popular storage technique on the server market. In order to design such systems, we

Permission to make digital or hard copies of all or part of this work for personal or classroom use is granted without fee provided that copies are not made or distributed for profit or commercial advantage and that copies bear this notice and the full citation on the first page. To copy otherwise, to republish, to post on servers or to redistribute to lists, requires prior specific permission and/or a fee.

SIMUTools 2010 March 15-19, Torremolinos, Malaga, Spain.

Copyright 2010 ICST, ISBN 78-963-9799-87-5. need adequate and simple simulation tools, which should be flexible enough to model dynamic environments including disk heterogeneity (several kind of disks), hierarchies and/or resource sharing.

A constant improvement in disk capabilities has been observed year after year [6]. However, many systems just ignore the disk heterogeneity and all disks are treated equally (as if they had same capacity and the lowest common speed). This results in not all potential capacity and performance gain is obtained.

We believe that it is very important to have simulation tools that allow researchers to compare the effects in the performance when a RAID system is ignoring the disk heterogeneity with the benefits of using the disks according to their capabilities. In addition, in order to design storage systems that can maximizes their capabilities, it is important to see the effects of using different data distribution policies as well as several user behavior (workloads) in the same comparisons.

However, the simulation process includes elements or files where the user could easily fail such as the binaries (the simulator), the configuration (the RAID that we want to simulate) and the workload (the user behavior that we want to simulate).

The errors are quite common in these files because all the simulation tools for RAID systems are only available on a Command Line Interface (CLI), which increases the human intervention that has been identified as a significant source of unmasked system failures [3]. When the comparatives are exhaustive, quite common in research projects, the failure probability in these elements is increased and the following problems could arise:

Delays for achieving results: The errors introduced by the Humans into the configurations and workload files result in a lot of wasted time in searching for the cause and solving of errors(on average one hour per error). Worst scenario is when these errors could induce failures in properly understanding the simulations results (we could think that a RAID system delivers good service times when it does not).

Recollection of results: when reporting simulation results by achieving and showing graphs, the human intervention 


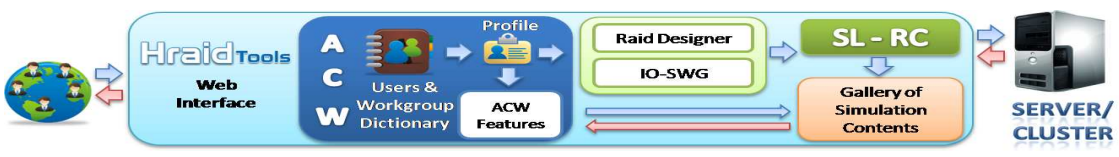

Figure 1: The workflow of HRaidTools suite showing the RAID-Designer, IO-SWG and SL-RC working with ACW.

again can introduce errors that modifies the simulation results.

On the other hand, the simulators are validated in a given architecture or format (little or big endian), have been tested in a operating system or configured for a compiler, so when the simulator is used in other environments, the simulation results can suffer variations and they may be not valid.

HRaid [7] already allows us simulating heterogeneous RAID systems in cluster of workstations but it is only available on a CLI and only includes the homogeneous data distributions RAID level 0,1 and 5 [8] and heterogeneous AdaptRaid [2] level 0 and 5. We propose HRaidTools, an on-line suite of tools based on HRaid that has been designed to reduce or even eliminate the simulation errors produced by the human intervention as well as the problems observed by the simulator when it is used in different environments. By using an Collaborative Work architecture of social networks profiles based on hierarchies, which avoids wasting time when accessing, handling, storing, organizing and searching the RAID configurations, workloads and results files. Finally, this suite includes several heterogeneous data distribution policies and redundancy levels unavailable in current RAID simulators.

\section{A SUITE OF SIMULATION TOOLS FOR HETEROGENEOUS RAID}

RAID-designer: We have implemented a simple shopping cart for designing RAID configurations, which avoids that users introduce not valid parameters to the configuration files and they can choose the disks, controllers, networks, data distributions and redundancy levels that are validated and available in the simulator.

The research job [4] was reproduced by using this tool and there were no errors in the RAID configuration files while that 52 errors (in mean one hour and half for solving each) were reported when using HRaid with a CLI.

IO-SWG: I/O synthetic workload generator. We have developed IO-SWG for generating I/O workload files that represent the users behavior using a given application, which can be used by our simulator without conflicts and errors. IO-SWG produces data for fields such as interarrival times, request size, position into the RAID and read/write ratio by using stochastic procedures following normal, poisson and uniform distributions. In addition, the generator also can produce interarrival times based on bursts (identified as a regular I/O behavior [5] ). This generator also can include several I/O traffics into the workload, which simulates the concurrency of several users in the same RAID.

SL-RC: Simulations Launcher and Results Collector. In order to launch simulations as well as recollect and show their results, we have implemented SL-RC, which includes a graph gallery showing mean/cumulative service times and throughput. All the simulations are launched on validated architectures for eliminating the portability problems (downloads and compilations of the simulator are not required). This guarantees the accurate of the simulation results and also eliminates the errors when reporting simulation results because the users cannot modify them.

ACW: Adaptive Collaborative Work. We have designed ACW as a web architecture based on profiles similar to the ones used in social networks but that is able to handle, independent and autonomous work groups, in hierarchy manner. The user can access to their results and graphs by using the ACW profiles of the HRaidTools webpage [1]. The ACW features are users management, contents organizer, task assignments and communication based on hierarchies. Figure 1 shows the workflow of the HRaidTools suite.

Adding New Data Distributions: We have implemented data distribution policies such as RAIDX or Pseudorandom policies (that are not available in other simulators) and we have designed strategies for testing them in heterogeneous RAID systems. We have performed the same for homogeneous distributions such as mirroring or chained declustering that now can be used on a set of heterogeneous disks.

\section{CONCLUSIONS}

We have presented HRaidTools: an on-line suite of simulation tools for heterogeneous RAID systems, which is focused on reducing the errors introduced by the humans and eliminating the portability problems in simulations. HRaidTools also includes IO-SWG for generating I/O workload files that can simulate several I/O traffics and bursty behavior. The suite makes availabble the configurations, workloads and results files by using the profiles provided by ACW architecture, which reduces the spent time when naming, storing, organizing and sharing the results of simulations. Finally, we have implemented new heterogeneous data distribution policies and redundancy levels unavailable in current RAID simulators.

\section{REFERENCES}

[1] Hraidtools demo. www.adaptivez.org.mx. user:HRaid paswd:HRaid.

[2] T. Cortes and J. L. Taking advantage of heterogeneity in disks arrays. $J P D C$, pages 448-464, 2003.

[3] A. G. D. Oppenheimer and D. Patterson. Why do internet services fail, and what can be done about it? USENIX, pages 109-116, 1988.

[4] J. Gonzalez and T. Cortes. Evaluating the effects of upgrading heterogeneous disk arrays. SPECTS06, pages 99-106, 2006.

[5] H. F. J. Zhang, A. S. Synthesizing representative i/o workloads for tpc-h. HPCA, pages 142-151, 2004.

[6] G. Patterson and Katz. A case for redundant arrays of inexpensive disks (raid). ACM SIGMOD ICMD., 2003.

[7] J. L. Toni Cortes. Hraid: A flexible storage-system simulator. ICPDPTA, pages 772-778, 1999.

[8] R. Yellin. The data storage evolution. has disk capacity outgrown its usefulness? Terada magazine, 2006. 